\title{
Article
}

\section{Tailored on demand anti-coagulant dosing: an in vitro and in vivo evaluation of 3D printed purpose-designed oral dosage forms}

Arafat, Basel, Qinna, Nidal, Cieszynska, Milena, Forbes, Robert Thomas and Alhnan, Mohamed A

Available at http://clok.uclan.ac.uk/22564/

Arafat, Basel, Qinna, Nidal, Cieszynska, Milena, Forbes, Robert Thomas ORCID: 0000-0003-3521-4386 and Alhnan, Mohamed A (2018) Tailored on demand anti-coagulant dosing: an in vitro and in vivo evaluation of 3D printed purpose-designed oral dosage forms. European Journal of Pharmaceutics and Biopharmaceutics, 128 . pp. 282-289. ISSN 0939-6411

It is advisable to refer to the publisher's version if you intend to cite from the work. http://dx.doi.org/10.1016/j.ejpb.2018.04.010

For more information about UCLan's research in this area go to http://www.uclan.ac.uk/researchgroups/ and search for < name of research Group>.

For information about Research generally at UCLan please go to http://www.uclan.ac.uk/research/

All outputs in CLoK are protected by Intellectual Property Rights law, including Copyright law. Copyright, IPR and Moral Rights for the works on this site are retained by the individual authors and/or other copyright owners. Terms and conditions for use of this material are defined in the policies page. 
1 Tailored on demand anti-coagulant dosing: an in vitro and in

2 vivo evaluation of 3D printed purpose-designed oral dosage

3 forms

4

5 Basel Arafat ${ }^{1,2}$, Nidal Qinna ${ }^{3}$ Milena Cieszynska ${ }^{1}$, Robert T Forbes ${ }^{1}$, Mohamed A 6 Alhnan $^{1^{*}}$

7

${ }^{1}$ School of Pharmacy and Biomedical Sciences and ${ }^{2}$ School of Medicine, University of Central Lancashire, Preston, Lancashire, UK.

${ }^{2}$ Faculty of Medical Sciences and Public health, Anglia Ruskin University, Chelmsford, UK

${ }^{3}$ Faculty of Pharmacy and Medical Sciences, University of Petra, Amman, Jordan.

*Corresponding author: MAlbedAlhnan@uclan.ac.uk

18

19

20

21

22

23

24 
Coumarin therapy has been associated with high levels of inter- and intra-individual variation in the required dose to reach a therapeutic anticoagulation outcome. Therefore, a dynamic system that is able to achieve accurate delivery of a warfarin dose is of significant importance. Here we assess, the ability of 3D printing to fabricate and deliver tailored individualised precision dosing using an in-vitro model. Sodium warfarin loaded filaments were compounded using hot melt extrusion (HME) and further fabricated via fused deposition modelling (FDM) 3D printing to produce capsular-ovoid-shaped dosage forms loaded at 200 and $400 \mu \mathrm{g}$ dose. The solid dosage forms and comparator warfarin aqueous solutions were administered by oral gavage to Sprague-Dawley rats. In vitro, warfarin release was faster at pH 1.2 in comparison to $\mathrm{pH}$ 2. A novel UV imaging approach indicated that the erosion of the methacrylate matrix was at a rate of 16.4 and $15.2 \mu \mathrm{m} / \mathrm{min}$ for horizontal and vertical planes respectively. In vivo, 3D printed forms were as proportionately effective as their comparative solution form in doubling plasma exposure following a doubling of warfarin dose (184\% versus $192 \%$ respectively). The 3D printed ovoids showed a lower $\mathrm{C}_{\max }$ of warfarin ( 1.51 and $3.33 \mathrm{mg} / \mathrm{mL}$ versus 2.5 and $6.44 \mathrm{mg} / \mathrm{mL}$ ) and a longer $T_{\max }(6$ and 3.7 versus 4 and $1.5 \mathrm{~h}$ ) in comparison to liquid formulation. This work demonstrates for the first time in vivo, the potential of FDM 3D printing to produce a tailored specific dosage form and to accurately titrate coumarin dose response to an individual patient.

Keywords: Rapid prototyping; Patient-centred; Personalized; Patient-specific; Three 


\section{Introduction}

For over 50 years now, coumarins have been the most prescribed oral anticoagulants.[1] Nevertheless, despite their wide use, coumarin therapy has been associated with a high level of inter-individual variation in dose required to achieve therapeutic anticoagulation response.[2] The administration of an inappropriate warfarin dose for example may place a patient in a hypercoagulable state or increase the patient's risk of bleeding complications early in therapy. As a consequence of over-anticoagulation response, there is an increased risk of major bleeding following the use of anticoagulants by $9.1 \%$ [3]. The American College of Chest Physicians (ACCP) supports an "induction" dose of 2 to $5 \mathrm{mg}$ per day which needs to be adjusted according to the patient's International Normalised Ratio (INR)[4]. The pharmacodynamics and pharmacokinetics of coumarins are largely influenced by many factors such as patient age, body weight, dietary vitamin $\mathrm{K}$ intake, concomitant medications, as well as various disease states.[2] Hence to ensure that the patient's INR remains within the target range, regular coagulation monitoring and dose modification is necessary.[5]

Nevertheless, limited doses of warfarin tablets are available in the market and dose modification usually requires multiple tablet ingestion or cutting or splitting of larger dose tablets, which could lead to variations in drug content.[6, 7] An area of potential improvement to warfarin therapy would be the ability to produce flexible on-demand precision tailored dose adjustments (particularly given warfarin's due to narrow therapeutic index). One technology that can potentially easily benefit anticoagulant therapy is 3D printing, owing to its flexible and precise manufacturing capability, which enables administration of the lowest effective dose of the drug to maintain the target INR. Indeed, recently, Vuddanda et al. (2017) demonstrated the potential of a re-engineered thermal inkjet printer to address the challenge of warfarin dosage personalisation, achieving highly reproducible minute warfarin dose of approximately $50 \mu \mathrm{g}$ $[8]$

3D printing potential and feasibility has been revealed in several fields such as aerospace, engineering, arts, as well as in fabricating medical implants and devices. Although still at its infancy in the field of personalised medicine, it is expected to revolutionise healthcare and set an innovative platform for pharmaceutical product design and extemporaneous preparation of patient-tailored dosage forms.[9] Fused deposition modelling (FDM) 3D printing, in particular, has been proposed as a platform for controlling the dose.[10] It has demonstrated its capability to manufacture mechanically stable tablets fabricated from pharmaceutical grade polymers without post-processing steps.[10-13] For instance, FDM 3D printing has been viably established using pharmaceutical grade polymers such as PVP [9, 14], methacrylate [15] and cellulose [12] based polymers.

The use of animal models is commonly used to predict formulation behaviour in humans. The use of rats in particular is favoured due to their small size, relatively low cost of breeding and up-keep, as well as the presence of large databases of drug pharmacokinetic data in rats and in humans.[16] Nevertheless, the testing of solid dosage forms in rats presents a challenge in terms of ease of administration. Owing to the need to use a small dosage form size, crushed tablets filled in capsule or suspended in liquid have often been used as an inferior alternative to test the in vivo performance of a tablet in rats.[17, 18] However, such approaches significantly alter the nature of the dosage form. More recently, the formulation of mini-tablets for animal use have been attempted $[19,20]$. It is therefore important to develop strategies that authentically test intact scaled down human dosage forms for animal studies to enable more reliable extrapolation of human pharmacokinetic responses. 
This work aimed to assess the suitability of FDM 3D printer technology for i) fabricating purposely designed solid dosage forms, and ii) tailoring the dose of a narrow therapeutic index drug, namely warfarin. To achieve this goal, rat-tailored FDM 3D printed warfarin ovoid tablets were printed and administered to Sprague-Dawley rats for testing to obtain their pharmacokinetics $(\mathrm{PK})$ parameters.

\section{Materials and methods}

\subsection{Materials}

Warfarin (sodium salt) was purchased from Arcos (UK). Eudragit E was donated from Evonik Industries (Darmstadt, Germany). Triethyl citrate (TEC) and tri-calcium phosphate (TCP) were supplied by Sigma-Aldrich (UK). Acetonitrile and methanol were supplied by British Drug Houses (BDH, London, UK). Scotch Blue Painter's tape $50 \mathrm{~mm}$ was supplied by 3M (Bracknell, UK).

\subsection{Preparation and optimisation of filaments}

In order to fabricate drug-loaded filaments, a hot melt extrusion method was implemented using a Thermo-Scientific HAAKE MiniCTW extruder (Karlsruhe, Germany). A 10 g sample of Eudragit E: TEC: TCP: sodium warfarin $46.75: 3.25: 49: 1)$ was accurately weighed and added gradually to counter flow twin-screw hot melt extruder, HAAKE MiniCTW (Karlsruhe, Germany). To allow homogeneous distribution of the powders, the molten mass was mixed in the extruder for at least $5 \mathrm{~min}$ prior to extrusion. The specific temperature of initial feeding and extrusion for the filament were 100 and $90{ }^{\circ} \mathrm{C}$ respectively. A torque control of $0.8 \mathrm{Nm}$ was used to extrude the filaments. Filaments were stored in sealed plastic bags at room temperature before $3 \mathrm{D}$ printing.

\subsection{Design and printing of tablets}

Tablets were constructed with the pre-prepared filaments using a MakerBot Replicator ${ }^{\circledR} 2 \mathrm{X}$ Experimental 3D Printer (MakerBot Industries, New York, USA) equipped with $0.4 \mathrm{~mm}$ nozzle size. The templates used to print the tablets were designed in a caplet shape using Autodesk $^{\circledR}$ 3ds Max ${ }^{\circledR}$ Design 2016 software version 18.0 (Autodesk, Inc., USA). The design was saved in a stereolithography (.stl) file format and was imported to the 3D printer's software, MakerWare Version 3.9.1.1143 (Makerbot Industries, LLC., USA).

Two sets of 3D printed tablets were fabricated:

In order to establish the ability of the system to control the low dose of drug for clinical use, a series of tablets with increasing volumes were then printed by increasing the dimensions of the design: length $\times$ width $\times$ heights $(\mathrm{L}, \mathrm{H}, \mathrm{W})$. The ratios between dimensions $(\mathrm{W}=\mathrm{H}=0.4 \mathrm{~L})$ remained constant. The size of the printed tablet $(M)$ was changed to achieve target doses of $0.5,1,3$ or $5 \mathrm{mg}$ (Table $1 \mathrm{~S}$ ).

To assess in vivo performance of this tablets in rats, a separate set of $3 \mathrm{D}$ printed ovoid shapes were manufactured with a cylindrical diameter of $2 \mathrm{~mm}$ and lengths of 5.5 or $11 \mathrm{~mm}$ to achieve a dose of 200 and $400 \mu \mathrm{g}$ respectively. Objects were printed using modified settings of the software as described earlier in our previous work at a temperature of $135{ }^{\circ} \mathrm{C}$. [15] 
Samples (raw materials, extruded filaments and printed tablets) were characterised using differential scanning calorimetry (DSC) and thermogravimetric analysis (TGA). For DSC analysis, a differential scanning calorimeter DSC Q2000 (TA Instruments, Elstree, Hertfordshire, UK) with a heating rate of $10^{\circ} \mathrm{C} / \mathrm{min}$ was used. Samples were heated to $100{ }^{\circ} \mathrm{C}$ for 5 min to exclude the effect of humidity then cooled to $-20{ }^{\circ} \mathrm{C}$. This was followed by a heat scan from $-20{ }^{\circ} \mathrm{C}$ to $300{ }^{\circ} \mathrm{C}$. Analysis was carried out under a purge of nitrogen $(50 \mathrm{~mL} / \mathrm{min})$. The data was analysed using TA 2000 analysis software. Standard $40 \mu \mathrm{L}$ TA aluminium pans and pin-holed lids were used with an approximate sample mass of $5 \mathrm{mg}$. All measurements were carried out in triplicate.

For TGA analysis, raw materials, extruded filaments and 3D printed tablets were analysed using a TGA/SDTA851e Mettler Toledo (Leicester, UK). Samples (5 mg, $\mathrm{n}=3$ ) were placed in $40 \mu \mathrm{L}$ aluminium pans and were then heated from 25 to $500^{\circ} \mathrm{C}$ at a heating rate of $10^{\circ} \mathrm{C} / \mathrm{min}$ and nitrogen gas flow of $50 \mathrm{~mL} / \mathrm{min}$. The thermal decomposition (or degradation) profile was analysed using STARE software version 9.00.

\subsection{X-ray powder diffraction (XRD)}

Samples (raw materials extruded filaments and printed tablets) were characterised using an X-ray diffractometer, D2 Phaser with Lynxeye (Bruker, Germany). Samples were scanned from $(2 \theta)=5^{\circ}$ to $50^{\circ}$ using $0.01^{\circ}$ step width and a 1 second time count. The divergence slit was $1 \mathrm{~mm}$ and the scatter slit $0.6 \mathrm{~mm}$. The wavelength of the X-ray was $0.154 \mathrm{~nm}$ using $\mathrm{Cu}$ source and a voltage of $30 \mathrm{kV}$. Filament emission was $10 \mathrm{~mA}$ using a scan type coupled with a theta/theta scintillation counter over $60 \mathrm{~min}$.

\subsection{Characterisation of tablet properties}

The hardness of six ovoid tablets was measured using a TBH 200 (Erweka GmbH, Heusenstamm, Germany). The mean crushing strength was determined, whereby an increasing force was applied to the tablet until it fractured or deformed.

In order to assess the friability of the tablets, 20 tablets were randomly selected, weighed and placed in a friability tester Erweka TAR 10 (Erweka GmbH, Heusenstamm, Germany) and the drum was then rotated at $25 \mathrm{rpm}$ for $4 \mathrm{~min}$. The tablets were reweighed and the differences in weight were calculated and displayed as a percentage of the original sample weight. In order to assess weight uniformity, 10 tablets were randomly selected and weighed. The average weights were measured and the percentage deviation of the individual tablets from the mean was determined.

To assess the impact of both HME and FDM 3D printing on drug content, 3 tablets from each formulation, were randomly selected and weighed. Tablets were then individually placed in a $1000 \mathrm{~mL}$ volumetric flask containing $0.1 \mathrm{M} \mathrm{HCl}$ and sonicated for $2 \mathrm{~h}$. The solutions were filtered through $0.22 \mu \mathrm{m}$ Millex-GP syringe filters (Merck Millipore, USA) and prepared for HPLC analysis.

Warfarin concentration in samples was assessed using an Agilent UV-HPLC 1260 series (Agilent Technologies, Inc., Germany) equipped with Kinetex C18 column $(100 \times 2.1 \mathrm{~mm}$, particle size $2.6 \mu \mathrm{m}$ ) (Phenomenex, Torrance, USA) and set at temperature $26{ }^{\circ} \mathrm{C}$. The mobile phase was 4:1 mixture of methanol: $\mathrm{pH} 3$ water (adjusted with orthophosphoric acid) at a flow rate of $1 \mathrm{~mL} / \mathrm{min}$. The injection volume was $100 \mu \mathrm{L}$ and the stop time was $10 \mathrm{~min}$. The 
wavelength was set to $230 \mathrm{~nm}$ and the retention time of the drug was 6.3 min with a limit of detection of $0.05 \mathrm{mg} / \mathrm{L}$.

\subsection{In vitro dissolution studies.}

a.Surface dissolution imaging. A Sirius SDi2, the second generation UV imaging system, designed to accommodate whole dosage forms, was used to visualize surface dissolution of sodium warfarin from the 3D printed dosage forms as a whole (Fig. 1). The 3D printed tablets were introduced into the SDi2's flow cell. The dissolution medium $\left(0.1 \mathrm{M} \mathrm{HCl}\right.$ at $\left.37^{\circ} \mathrm{C}\right)$ applied at a flow rate of $8.2 \mathrm{~mL} / \mathrm{min}$. The dissolution medium was introduced into the flow cell in the open loop configuration, from bottom to top, with an equivalent linear velocity of $1 \mathrm{~cm} / \mathrm{min}$. Dissolution experiments were recorded for a total duration of $60 \mathrm{~min}$. The two dimensional detection area on the SDi2 is significantly larger than for the SDI $(24 \mathrm{~mm}$ width $\mathrm{x} 28 \mathrm{~mm}$ height) to accommodate dissolution imaging profiling of intact whole dosage forms, with a spatial resolution of $13.75 \mu \mathrm{m}$. The flow cell was illuminated using alternate pulses from two 255 and $520 \mathrm{~nm}$ wavelength LEDs. The dual wavelength enables two separate video captures to be produced from a single experiment. Real-time data were then used to measure and differentiate between drug release into solution and tablet erosion from the 255 and $520 \mathrm{~nm}$ light obtained videos, respectively.

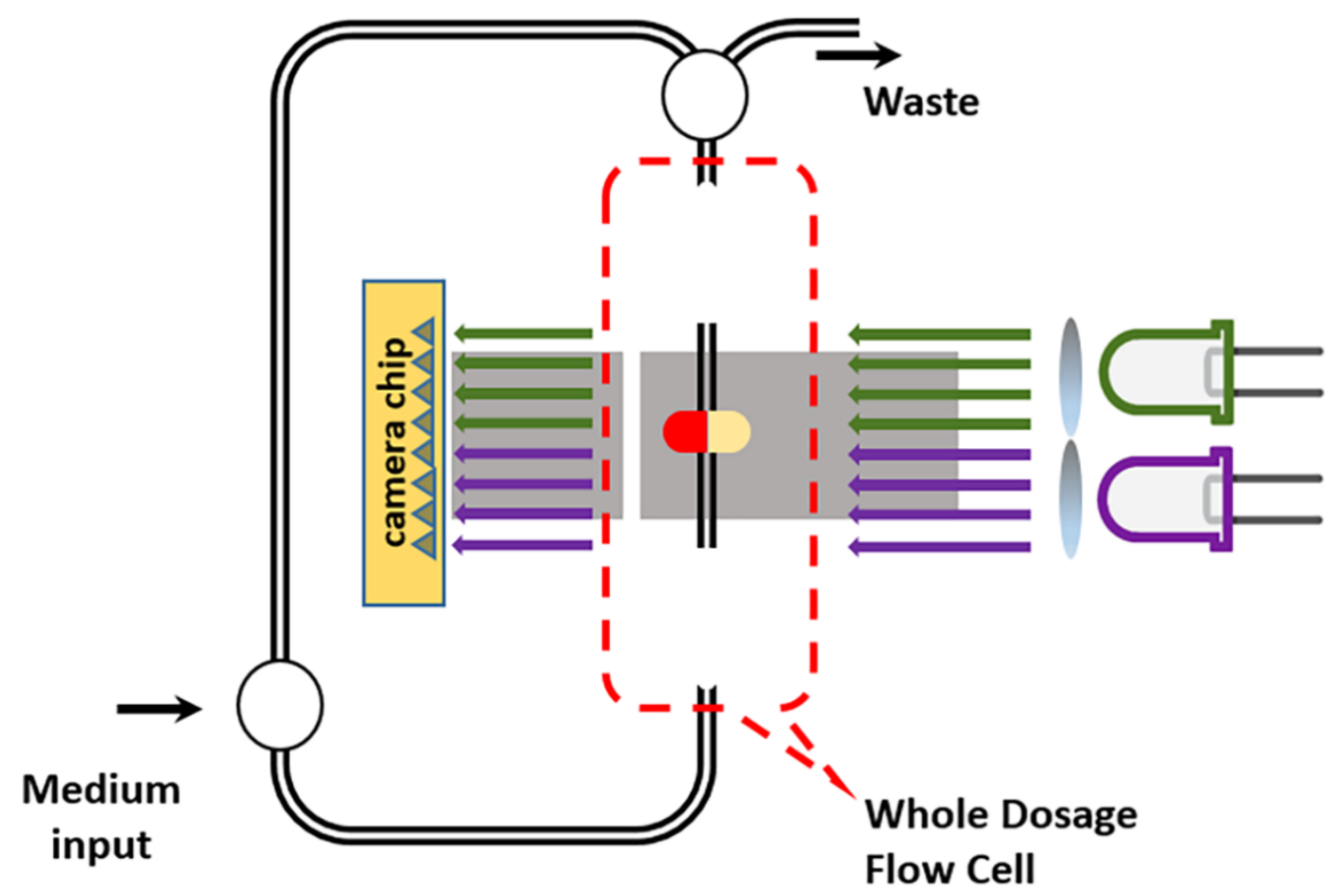

Figure 1. Schematic diagram of SDi2 instrument. LED's of different wavelength are em ployed to illuminate the 3D printed tablet in flow through cell filled with gastric medium. The obscuration or absorbance of the sample was recorded using an Actipix detector. The medium is pre-heated to $37^{\circ} \mathrm{C}$ before going through the Whole Dosage Flow Cell and is recirculated in a closed loop configuration. investigated using a USP II Erweka DT600 dissolution tester (Erweka GmbH, Heusenstamm, 
Germany). Three tablets were randomly selected and individually placed in the dissolution vessels each containing $900 \mathrm{~mL}$ of a fasted state simulated gastric fluid (FaSSGF) $(1.75 \mathrm{mM}$ SLS, $0.01 \mathrm{~N} \mathrm{HCl}, 0.2 \% \mathrm{NaCl}, \mathrm{pH} 2.0)$ at $50 \mathrm{rpm}$ and $37 \pm 0.5^{\circ} \mathrm{C}$. Aliquots $(5 \mathrm{~mL})$ were manually collected using $5 \mathrm{~mL}$ Leur-Lock syringes at $0,5,10,15,20,25,30,40,50$ and $60 \mathrm{~min}$ time intervals and filtered through an Agilent $0.22 \mu \mathrm{m}$ filter. Each aliquot withdrawn was replaced with $5 \mathrm{~mL}$ of $0.1 \mathrm{M} \mathrm{HCl}$ and analysed using the above described HPLC method.

\subsection{In vivo studies}

Adult heathy male Sprague-Dawley rats with an average weight of $240 \pm 15 \mathrm{~g}$ accommodated at the University of Petra's Animal House (Amman, Jordan) under controlled temperature $\left(22{ }^{\circ} \mathrm{C}-24{ }^{\circ} \mathrm{C}\right)$, humidity $(55 \%-65 \%)$, and a 12 hours photoperiod cycle. All rats were acclimatized for 10 days before experimentation. Rats were weighed and randomized into groups ( $\mathrm{n}=6$ rats per cage). Rats were offered standard pellet diet (Jordan Feed Company Ltd., Amman, Jordan) and served clean tap water ad libitum. However, animals were fasted for 18 hours before the day of testing. All experiments were carried out in accordance with University of Petra's Institutional Guidelines on Animal Use that adopts the guidelines of the Federation of European Laboratory Animal Sciences Association (FELASA). The animal study protocols were revised and approved by the Higher Research Council at the Faculty of Pharmacy and Medical Sciences, University of Petra (Amman, Jordan).

3D printed tablets ( 200 or $400 \mu \mathrm{g}$ ) were administered to the rats via any oral capsule stainless steel feeding needle. Comparison control $1 \mathrm{~mL}$ warfarin solutions (200 or $400 \mu \mathrm{g}$ ), equivalent to the tablet doses, were freshly prepared and administered to the rats by a stainless steel oral gavage needle (Harvard Apparatus, Kent, UK). Following oral administrations, blood samples were pooled from rat's tail ( $\mathrm{n}=6$ rats per group) at different time intervals namely at; $1,2,3,4$, 6 and 8 hours post administration. Blood was left to clot, centrifuged for $10 \mathrm{~min}$ at 2000G, and then serum was separated and transferred directly into Eppendorf tubes, and kept in a freezer at $-20{ }^{\circ} \mathrm{C}$ until analysis.

\subsection{Analysis of warfarin}

For the analysis of warfarin an MS/MS system: API 3200 (Applied Biosystems, MDS SCIEX, USA) attached to Agilent 1200 HPLC (Agilent Technologies, USA) controlled by Analyst 1.6.1 software, was utilised. For the extraction of warfarin from the samples, $100 \mu \mathrm{L}$ of spiked/blank plasma were pipetted into previously labelled Eppendorf tube, $25 \mu \mathrm{L}$ of the internal standard (IS) Fenofibric acid (FFA) from $100.0 \mu \mathrm{g}$ FFA/mL IS solution was added to the tubes and vortexed for $30 \mathrm{sec}$. Afterwards, the precipitation solution, acetonitrile $(400.0 \mu \mathrm{L})$ was added to the tube and vortexed for further $1 \mathrm{~min}$. Samples were then centrifuged for $5 \mathrm{~min}$ at 14,000 rpm and the supernatant was collated and transferred into an auto-sampler micro vial for analysis. The mobile phase used for analysis comprised of (30:70) mixture of ammonium chloride $0.001 \mathrm{M}$ : acetonitrile respectively eluted at a flow rate of $0.7 \mathrm{~mL} / \mathrm{min}$ through a Thermo BDS Hypersil C18 $(50 \times 2.1 \mathrm{~mm}$, particle size $5 \mu \mathrm{m})$ column (Thermo Fisher Scientific, Germany) at the temperature $30^{\circ} \mathrm{C}$. The injection volume was $5 \mu \mathrm{L}$ and the stop time was $0.7 \mathrm{~min}$. The retention time of the drug was $0.3 \mathrm{~min}$ with a limit of detection of $10 \mathrm{ng} / \mathrm{mL}$.

\subsection{Statistical Analysis}

Independent sample T-test was also employed using a SPSS Software (22.0.0.2) to analyse the in vitro tablet characterisation results. Differences in results where $p \leq 0.05$ were considered significant. 


\section{Results and discussion}

In this study, we explored the adaptability of FDM based 3D printing to engineer and control the dose of immediate release warfarin tablets. When a series of warfarin tablets with increasing dimensions were printed (Fig. 2A, Table S1), a high level of correlation was identified between the theoretical volume of the tablet design and their weights $\left(\mathrm{R}^{2}=0.9934\right)$ (Fig. 2B). This indicated the ability of FDM 3D printing method to achieve a sufficient control of the mass of 3D printed tablets. To establish the ability of such 3D printing method to control dosage, theoretical doses based on tablet mass and measured dose of warfarin in the tablet were compared. The range of dose accuracy was between $91.5 \%$ and $102.4 \%$ (Fig. 2C). The coefficient of determination between target and achieved dose $\left(\mathrm{R}^{2}=0.9902\right)$ showed that it is possible to fabricate tablets with desired dose of warfarin through volume modification even at a minute dose of $500 \mu \mathrm{g}$ (Fig. 2D). With the advances in 3D printers, additional safeguards and quality control mechanisms can be introduced to the evolving technology [21], which are expected to minimise dose variation in the near future.
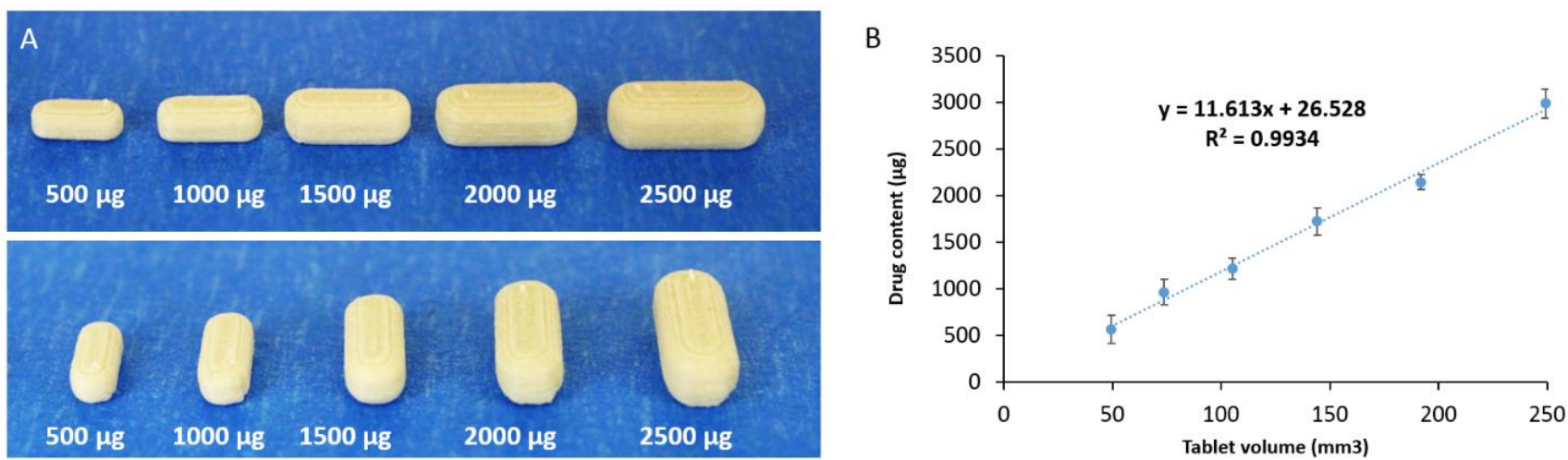

C

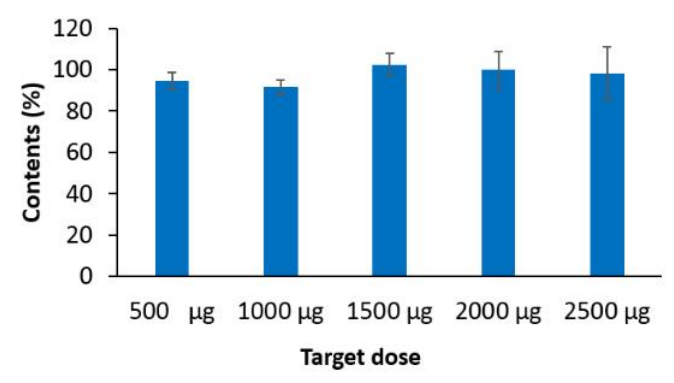

D

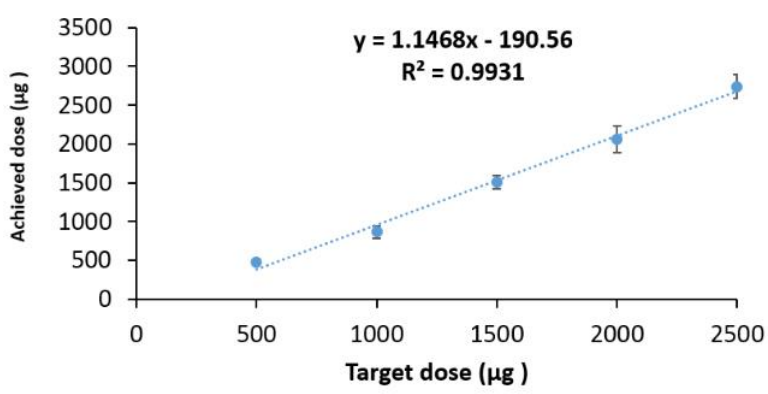

Figure 2. Precision of $3 D$ printing to control low dose sodium warfarin. (A) Images of warfarin loaded FDM 3D printed tablets with increasing dose, (B) Correlation between the theoretical volume and tablet mass, (C) warfarin dose accuracy in the 3D printed tablets and (D) correlation between theoretical volume and warfarin dose $(n=3, \pm S D)$.

Profiles from thermogravimetric analyses of warfarin and other additives as well as HME processed filaments and 3D printed tablets are shown if Fig. 3A. Sodium warfarin alone or incorporated in filaments did not suffer a significant weight loss at the printing temperature $135^{\circ} \mathrm{C}$. Therefore, it can be assumed that minimal or no degradation of warfarin occurs in the HME as well as in the FDM's nozzle under the utilised temperatures (Fig. 3A). The processing temperatures were lower than the melting point of sodium warfarin $\left(161{ }^{\circ} \mathrm{C}\right)$. Differential scanning calorimetry was also conducted to examine the plasticising effect of components on the methacrylic filament. As demonstrated in Fig 3B, the addition of TEC as a plasticizer significantly depressed the $\mathrm{Tg}$ of filament to $34^{\circ} \mathrm{C}$ from $54^{\circ} \mathrm{C}$. However, warfarin was found 
to have no significant effect on the Tg of Eudragit E. This could be attributed to the minute percentage of the drug used in the polymeric structure $(1 \% \mathrm{w} / \mathrm{w})$, which was insufficient to significantly influence the mobility of methacrylic polymer chains within the filament matrix. XRD spectra showed that $\beta$-calcium tribasic phosphate displayed peaks at 2 -theta $=17^{\circ}, 27.8^{\circ}$, $31^{\circ}, 34.4^{\circ}$ corresponding to calcium tribasic phosphate [22], whilst warfarin drug substance showed peaks at 2-theta $=12.4^{\circ}$ and $18^{\circ}$. XRD spectra of the warfarin filament and tablet showed an absence of these specific peaks $[23,24]$, suggesting the warfarin is present in an amorphous form within the tablet structure (Fig. 3C).

From determination of the mechanical properties of the 3D printed tablets, the friability of all batches was found to be zero percent. This highlights a prime advantage of FDM 3D printing in generating mechanically stable tablets over its rival technologies such as extrusion 3D printing [25] and powder-based 3D printing. [26, 27] The lack of a drying step or any postprinting finishing procedures, clearly demonstrates the potential of this technology to instantly produce a ready-to-use dosage form within minutes following a healthcare team request.
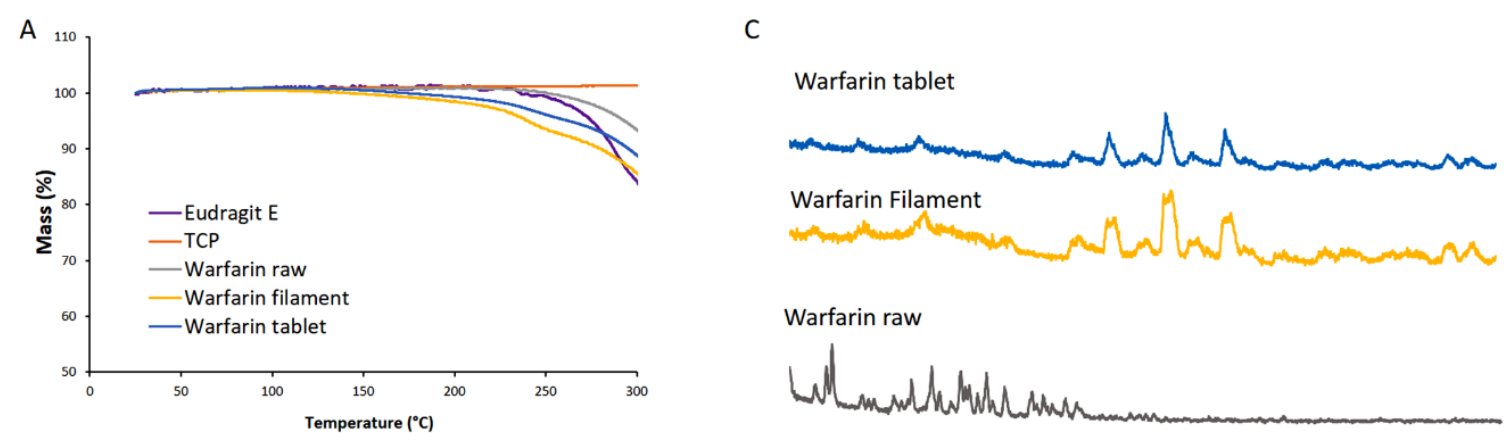

B
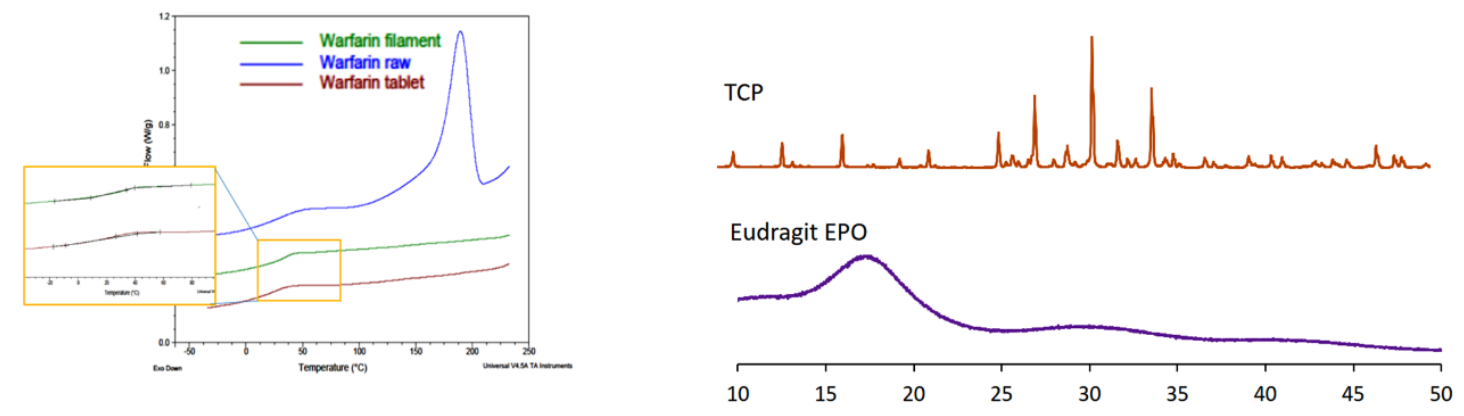

Figure 3. Thermal analysis of Eudragit $E$ based 3D printing filaments. (A) Thermal degradation profiles for Eudragit E, sodium warfarin, TCP, warfarin loaded filament and tablet, (B) DSC thermograph for warfarin loaded filament and tablet, (C) XRD spectra of Eudragit E, TCP, warfarin, and warfarin loaded filament and tablet.

The release pattern of warfarin from the methacrylic matrix was investigated using a modified FaSSGF [28] as a dissolution medium (Fig. 4). All tablets showed a release pattern of $>80 \%$ dissolution at $45 \mathrm{~min}$ regardless of their individual sizes. The dissolution release profile was attributed to the ionisation of the amino groups of the cationic methacrylic polymer in modified FaSSGF ( $\mathrm{pH} 2.0$ ), which leads to electrostatic repulsion between cationic polymer chains and facilitates polymer dissolution and drug release. The release was compliant with 


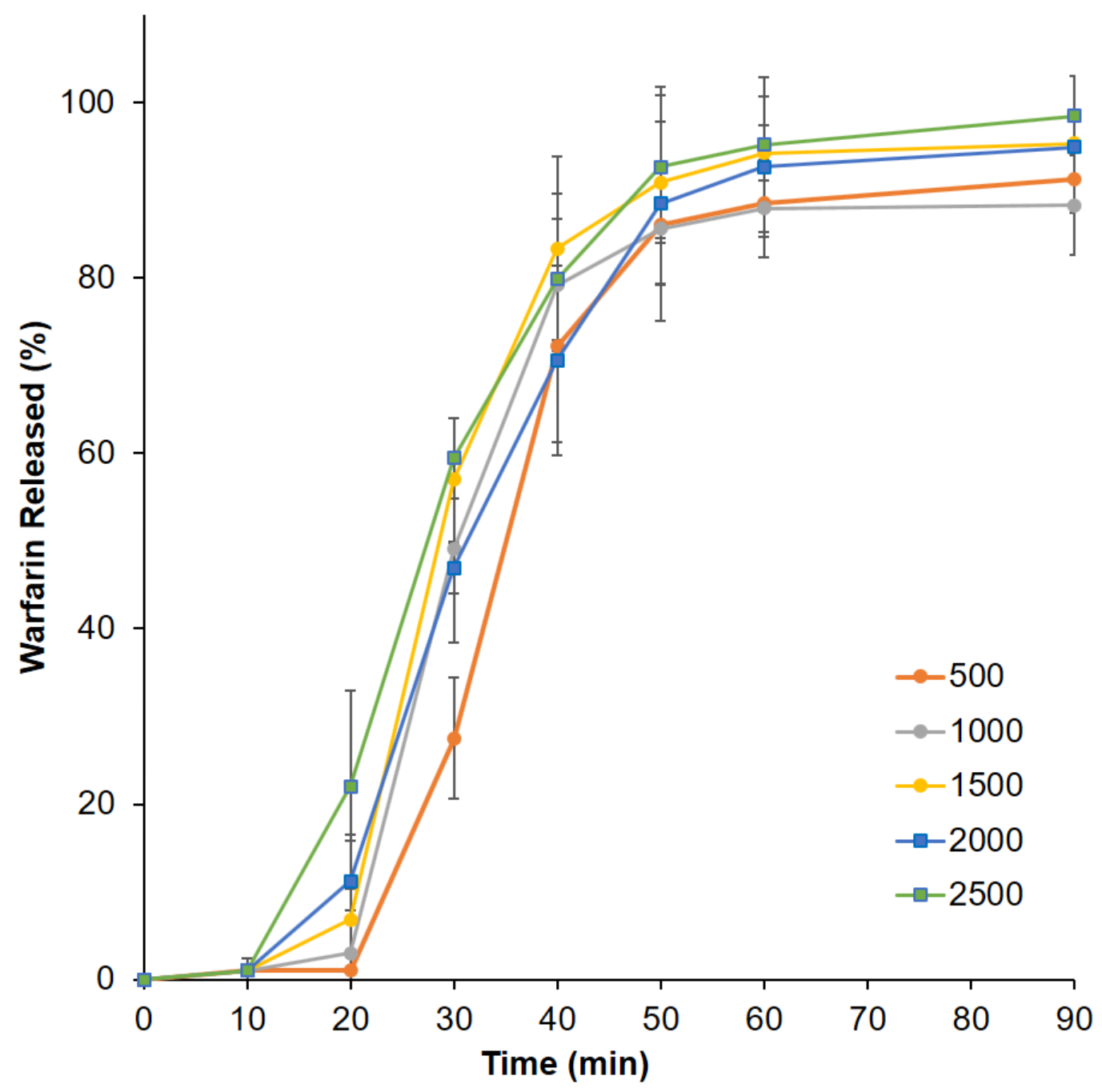

Figure 4. In vitro release pattern of sodium warfarin from 3D printed tablets of different doses from a USPII dissolution test in modified FaSSGF $(p H 2.0)(n=3, \pm S D)$.

To better understand the drug release from the 3D printed tablets, the dissolution behaviour of the tablets at the dissolving surface in contact with the dissolution media was explored. A single wavelength system has been previously used to study drug powder dissolution [30]. Here we employ a UV imaging technology capable of generating visual images from simultaneous spectroscopic evaluation for a complete dosage form (Fig. 5A, B). A clear advantage of using such a novel UV-VIS imaging technique over the other well-established imaging techniques lies in the simplicity of operation and interpretation of generated data, analogous to findings by Østergaard.[31] The measurement of light intensity passing through an area of a quartz tube as a function of position and time can also enable quantification of the drug substance at different time intervals. During the dissolution process, drug concentration increased in the first $20 \mathrm{~min}$ in the closed loop of the flow-through system. Simultaneously the tablet size was eroded at a rate of 16.4 and $15.2 \mu \mathrm{m} / \mathrm{min}$ for horizontal and vertical planes respectively. It is worth noting that surface analysis indicated no significant swelling in the first $5 \mathrm{~min}$. The simultaneous drug release data suggested that under the dissolution conditions of study, the majority of drug release took place by a diffusion mechanism before the erosion of the methacrylic matrix within the flow-through cell is complete. 

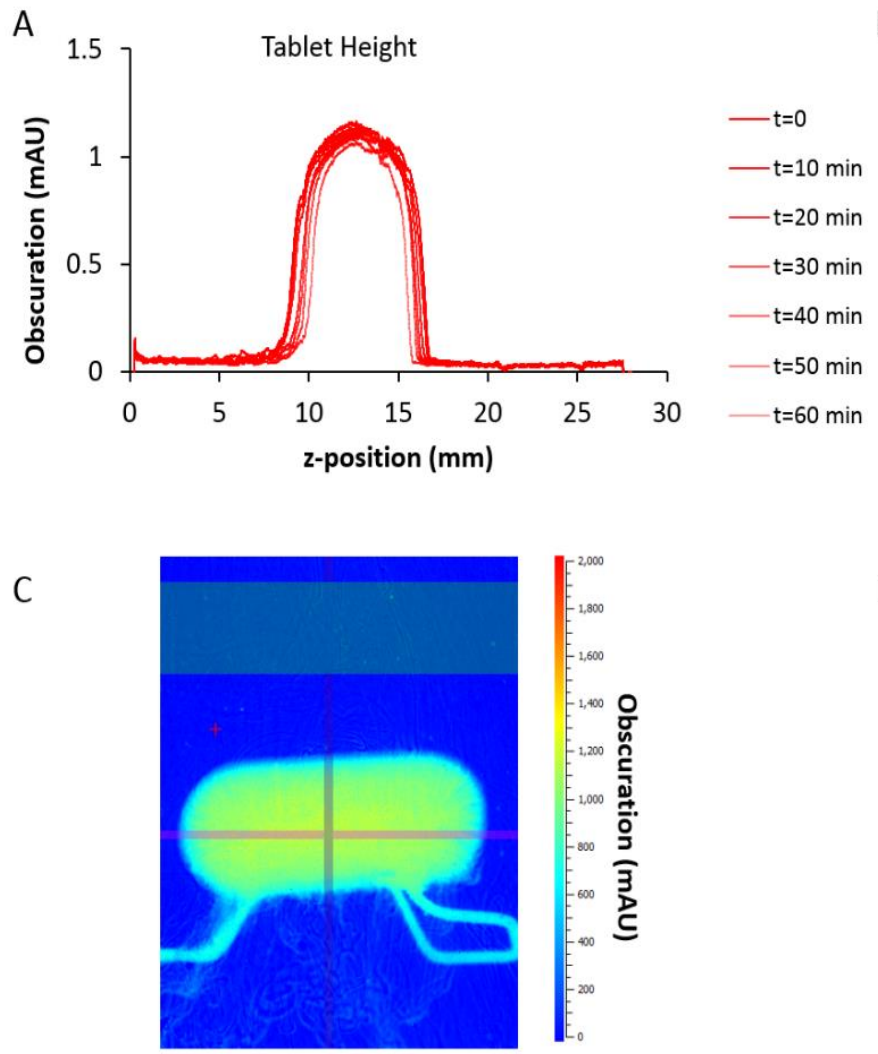

B
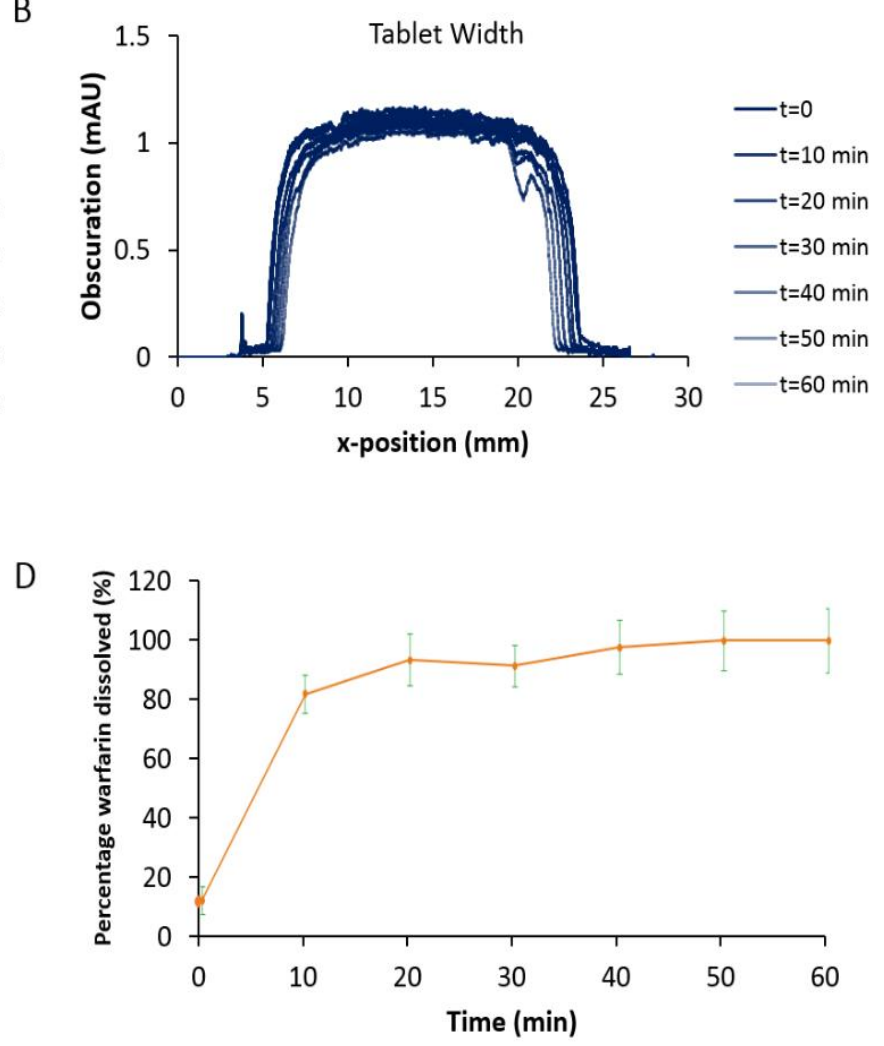

Figure 5. Changes in tablet height (A) and width (B) at 0, 10, 20, 30, 40, 50 and 60 min of the flow through dissolution test using Actipix SDI 2 dissolution imaging technology. (C) UV absorbance image following the illumination of follow cell containing warfarin 3D printed tablet at $255 \mathrm{~nm}$ wavelength. (D) Percentage sodium warfarin release from 3D printed tablet during dissolution test $(n=3, \pm S D)$.

A prime advantage of 3D printing technologies lies in their highly flexible nature and capacity to construct dosage forms with accurate spatial distribution of ingredients compared to traditional manufacturing techniques. Therefore, constructs can now be printed to suit the anatomy of not only a particular animal but according to the weight and size of that subject. Rats are commonly considered most suitable for determining the mechanism of drug absorption and bioavailability values from powder or solution formulations [32] as well as micro- or nanoparticles [33].

Two different warfarin tablets were specially designed (Fig. 6A1) to mimic the dimensions of commonly used hard capsules intended for oral delivery to rats. Tablets were successfully printed (Fig. 6A2) and were orally gavaged to rats. The pharmacokinetic parameters of warfarin following oral administration either as 3D printed tablets or in a solution form were evaluated (Table 1, Fig. 6B, C). Warfarin plasma exposure was significantly different when an equal dose was administered either as solutions or as $3 \mathrm{D}$ printed tablets. The solution showed a markedly higher $\mathrm{C}_{\max }(2.5$ and $6.44 \mathrm{mg} / \mathrm{mL})$ and shorter $\mathrm{T}_{\max }(2.67$ or $1.5 \mathrm{~h})$ for the 200 or $400 \mu \mathrm{g} / \mathrm{mL}$ solution respectively, in comparison to $C_{\max }$ values $\left(1.51\right.$ and $3.33 \mathrm{mg} / \mathrm{mL}$ ) and $T_{\max }$ values $(6$ or $3.7 \mathrm{~h})$ for $200 \mu \mathrm{g}(p<0.05)$ and $400 \mu \mathrm{g}(p<0.01)$ warfarin tablets respectively. 
Table 1. Summary of pharmacokinetic parameters of warfarin following oral gavage of 200 or $400 \mu \mathrm{g}$ from sodium warfarin solution and 3D printed tablets to adult heathy male Sprague-Dawley rats.

\begin{tabular}{|c|c|c|c|}
\hline Dose & $\operatorname{Cmax}^{*}(\mu \mathrm{g} / \mathrm{mL})$ & $\operatorname{Tmax}^{*}(h)$ & $\begin{array}{l}\operatorname{AUC}_{1-8}^{*} \\
\text { (mg/mL.h) }\end{array}$ \\
\hline Solution $(200 \mu \mathrm{g})$ & $2.5 \pm 0.3$ & $2.67 \pm 1.15$ & $20.64 \pm 1.9$ \\
\hline Solution $(400 \mu g)$ & $6.44 \pm 0.1$ & $1.5 \pm 0.6$ & $39.56 \pm 7.4$ \\
\hline 3D printed tablet $(200 \mu \mathrm{g})$ & $1.51 \pm 0.09$ & $6 \pm 1.6$ & $10.8 \pm 2$ \\
\hline 3D printed tablet $(400 \mu \mathrm{g})$ & $3.33 \pm 0.5$ & $3.7 \pm 1$ & $19.93 \pm 1$ \\
\hline
\end{tabular}

${ }^{\circ} \mathrm{C}_{\max }$, Maximum serum concentration; $\mathrm{T}_{\max }$, Time at which $\mathrm{C}_{\max }$ is observed; and $\mathrm{AUC}_{1-8}$, area under curve.
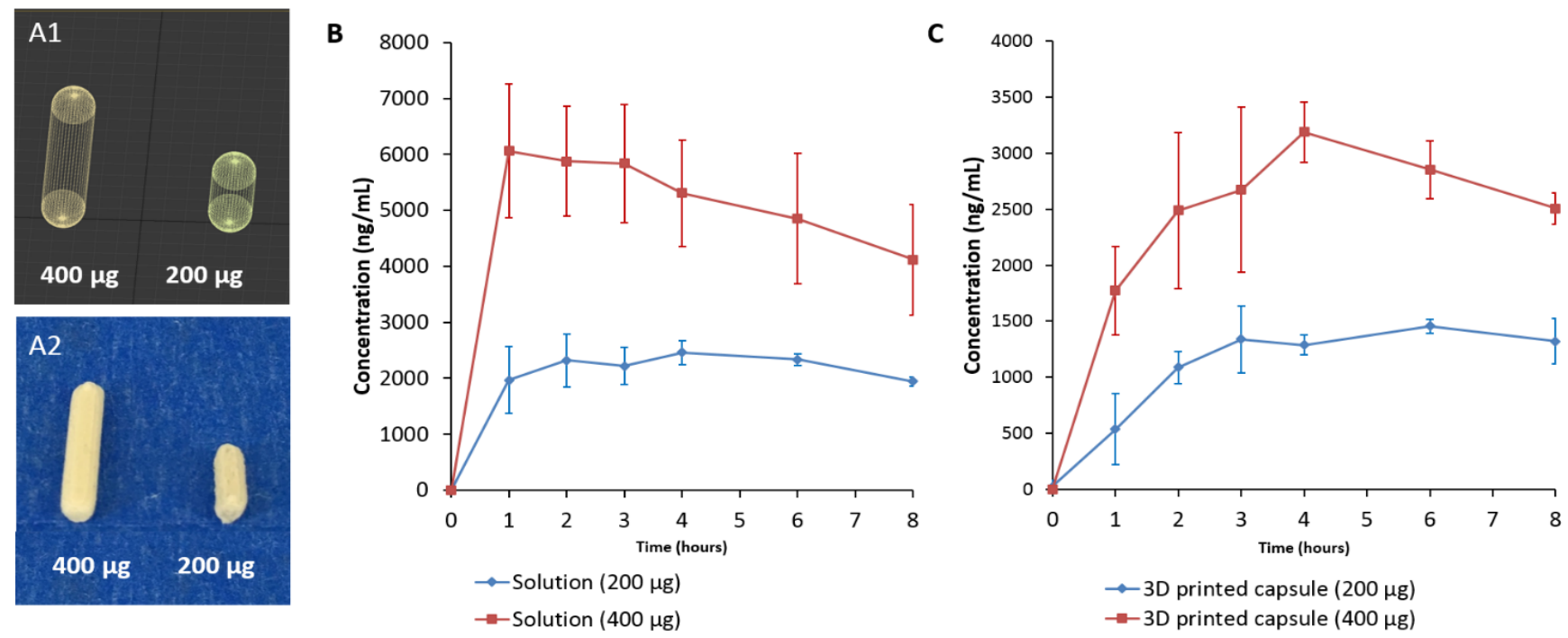

Figure 6. (A1) Rendered images and (A2) photographs of purpose designed 3D printed tablets for oral gavage in rats, (B) Plasma concentration- time profile of warfarin following the oral dosing of 200 or $400 \mu \mathrm{g}$ from (B) warfarin solution and (C) warfarin loaded 3D printed tablets to adult heathy male Sprague-Dawley rats $(n=4)$, error bars $\pm S D$.

Contributing to the finding above, the additional erosion step of Eudragit $\mathrm{E}$ in the $3 \mathrm{D}$ printed tablets is thought to slow down the release of warfarin from the tablets.. In reality, in an in vivo situation, dissolution is expected to be slower than suggested by in vitro dissolution techniques since a significantly higher $\mathrm{pH}$ of the stomach contents in rats $\mathrm{pH} 3.2$ (fed) and $\mathrm{pH} 3.9$ (fasted) [34] exists compared to the in vitro human simulation media conditions. Furthermore, the low fluid volume $(3.2 \pm 1.8 \mathrm{~mL})$ in the fasted rats are likely to contribute to slower dissolution rates of the methacrylate polymer in vivo than in vitro. The longer $\mathrm{T}_{\max }$ of the tablets might also be attributed to the slower transit time of the relatively large oral units in rodents as previously observed to be the case for oral pellets. Such effects are likely to be minimal in healthy human adults where greater volumes of gastric fluids [35, 36] and a lower $\mathrm{pH}[37]$ at fasted state are known. In summary, when extrapolating the findings to the human situation, it should be considered that such delay has been augmented by the slower erosion of cationic polymer is rat gastric environments rats due to their relatively higher gastric $\mathrm{pH}$ and lower fluid contents 
(yielded by FDM 3D printing) is that they match the release from standard compressed powdered tablets. The data we present suggests that dissolution of $3 \mathrm{D}$ tablets requires acceleration. However recently, there has been reports of utilizing 3D printer geometry to fabricate tablet with complex structure to accelerate drug release [38, 39].

On the other hand, 3D printed tablets were proportionately effective as solution formulations, in that a doubling of warfarin dose from the either tablet or solution resulted in a rough doubling of measured plasma exposure with $\mathrm{AUC}_{1-8}$ values doubling from $20.64 \pm 1.9$ to $39.56 \pm 7.4 \mu \mathrm{g} / \mathrm{mL}$ for the 200 and $400 \mu \mathrm{g} / \mathrm{mL}$ solutions respectively and from $10.8 \pm 2$ to $19.93 \pm 1 \mu \mathrm{g} / \mathrm{mL}$ for the 200 and $400 \mu \mathrm{g}$ 3D printed capsules, respectively (184\% versus $192 \%$ respectively). Envisioning a future scenario, a healthcare staff member may be able to use computer software to digitally directly tailor and manufacture an individualised precision dose and consequently provide plasma levels of warfarin appropriate to an individual patient's need.

In summary, the findings in this study clearly demonstrate the potential of $3 \mathrm{D}$ printing as a platform to design animal-suitable solid dosage forms and thus in principle provide a pathway for human use with the potential advantage of digitally titrating an individuals dose in response to clinical data. We have also shown the utility of a novel dissolution imaging system to give mechanistic insights into the dissolution process of a 3D-printed tablet dosage form. 


\section{Conclusions}

This study demonstrates the flexibility of FDM 3D printers to fabricate solid dosage forms to purposely suit the anatomy of an animal subject. UV imaging indicated that the erosion of methacrylic matrix takes place at 16.4 and $15.2 \mu \mathrm{m} / \mathrm{min}$ for horizontal and vertical planes respectively and resulted in delayed plasma exposure in comparison to warfarin solutions. Moreover, the titration of dose of a narrow therapeutic index drug, warfarin, has been demonstrated in vitro and in vivo. In principle, the technology holds the promise to provide a much more dynamic and responsive anticoagulant regime to suit a constantly changing patient's INR profile. Such an approach can provide patients with a safer, more accurate and computerised alternative to the more commonly used approach of dosing using multiple tablets to include tablet splitting.

\section{Acknowledgments}

393 The authors would like to thank UCLAN Innovation Team for this support and Mrs Reem 394 Arafat for her help with graphics design.

395 Conflicts of interest M A Alhnan is the innovator in patent applications WO 2016038356

396 A1, WO2017072536A1 and WO2018020237A1 in the field of 3D printing of medicines. 
[1] K.A. Bauer, Pros and cons of new oral anticoagulants, ASH Education Program Book, 2013 (2013) 464-470.

400

[2] H. Takahashi, H. Echizen, Pharmacogenetics of CYP2C9 and interindividual variability in anticoagulant response to warfarin, Pharmacogenomics J, 3 (2003) 202-214.

[3] L.A. Linkins, P.T. Choi, J.D. Douketis, Clinical impact of bleeding in patients taking oral anticoagulant therapy for venous thromboembolism: a meta-analysis, Ann Intern Med, 139 (2003) 893-900.

405 anticoagulants: mechanism of action, clinical effectiveness, and optimal therapeutic range, Chest, 119 (2001) 8S-21S.

408

[5] M. Kuruvilla, C. Gurk-Turner, A review of warfarin dosing and monitoring, Proceedings (Baylor University. Medical Center), 14 (2001) 305-306. uniformity for half-tablets of 6 commonly split medications, J Manag Care Pharm, 15 (2009) 253-261.

[7] J.E. Polli, S. Kim, B.R. Martin, Weight uniformity of split tablets required by a Veterans Affairs policy, J Manag Care Pharm, 9 (2003) 401-407. [8] P.R. Vuddanda, M. Alomari, C.C. Dodoo, S.J. Trenfield, S. Velaga, A.W. Basit, S. Gaisford, Personalisation of warfarin therapy using thermal ink-jet printing, Eur J Pharm Sci, 117 (2018) 80-87. Temperature FDM 3D Printing for the Manufacture of Patient-Specific Immediate Release Tablets, Pharm Res, 33 (2016) 2704-2712.

[10] J. Skowyra, K. Pietrzak, M.A. Alhnan, Fabrication of extended-release patient-tailored prednisolone tablets via fused deposition modelling (FDM) 3D printing, Eur J Pharm Sci, 68 (2015) 11-17.

[11] A. Goyanes, P. Robles Martinez, A. Buanz, A.W. Basit, S. Gaisford, Effect of geometry on drug release from 3D printed tablets, Int J Pharm, 494 (2015) 657-663.

[12] K. Pietrzak, A. Isreb, M.A. Alhnan, A flexible-dose dispenser for immediate and extended release 3D printed tablets, Eur J Pharm Biopharm, 96 (2015) 380-387. [13] A. Goyanes, H. Chang, D. Sedough, G.B. Hatton, J. Wang, A. Buanz, S. Gaisford, A.W. Basit, Fabrication of controlled-release budesonide tablets via desktop (FDM) 3D printing, Int J Pharm, 496 (2015) 414-420.

[14] T.C. Okwuosa, B.C. Pereira, B. Arafat, M. Cieszynska, A. Isreb, M.A. Alhnan, Fabricating a Shell-Core Delayed Release Tablet Using Dual FDM 3D Printing for Patient-Centred Therapy, Pharm Res, 34 (2017) 427-437.

[15] M. Sadia, A. Sosnicka, B. Arafat, A. Isreb, W. Ahmed, A. Kelarakis, M.A. Alhnan, Adaptation of pharmaceutical excipients to FDM 3D printing for the fabrication of patient-tailored immediate release tablets, Int J Pharm, 513 (2016) 659-668.

437 [16] X. Cao, S.T. Gibbs, L. Fang, H.A. Miller, C.P. Landowski, H.C. Shin, H. Lennernas, Y. Zhong, 438 G.L. Amidon, L.X. Yu, D. Sun, Why is it challenging to predict intestinal drug absorption and oral bioavailability in human using rat model, Pharm Res, 23 (2006) 1675-1686.

[17] D. Mann, US Patent 4637816 A: Apparatus for the oral administration of capsules to animals, in, 1987. 

Alternative method of oral dosing for rats, J Am Assoc Lab Anim Sci, 49 (2010) 335-343. [19] A. Vetter, G. Perera, K. Leithner, G. Klima, A. Bernkop-Schnurch, Development and in vivo bioavailability study of an oral fondaparinux delivery system, Eur J Pharm Sci, 41 (2010) 489497.

[20] J.Y. Kim, H.J. Bae, J. Choi, J.R. Lim, S.W. Kim, S.H. Lee, E.S. Park, Efficacy of gastro-retentive forms of ecabet sodium in the treatment of gastric ulcer in rats, Arch Pharm Res, 37 (2014) 1053-1062. [21] N. Sandler, I. Kassamakov, H. Ehlers, N. Genina, T. Ylitalo, E. Haeggstrom, Rapid interferometric imaging of printed drug laden multilayer structures, Sci Rep, 4 (2014) 4020. [22] Brian R. Genge, Licia Wu, Glenn R. Sauer, Roy E. Wuthier, R. Genge, US Patent 7527687 B2 Biocompatible cement containing reactive calcium phosphate nanoparticles and methods for making and using such cement., in, 2009. [23] A. Nguyenpho, A.B. Ciavarella, A. Siddiqui, Z. Rahman, S. Akhtar, R. Hunt, M. KorangYeboah, M.A. Khan, Evaluation of In-Use Stability of Anticoagulant Drug Products: Warfarin Sodium, J Pharm Sci, 104 (2015) 4232-4240. [24] Z. Rahman, M. Korang-Yeboah, A. Siddiqui, A. Mohammad, M.A. Khan, Understanding effect of formulation and manufacturing variables on the critical quality attributes of warfarin sodium product, Int J Pharm, 495 (2015) 19-30.

[25] S.A. Khaled, J.C. Burley, M.R. Alexander, C.J. Roberts, Desktop 3D printing of controlled release pharmaceutical bilayer tablets, Int J Pharm, 461 (2014) 105-111. [26] D.-G. Yu, C. Branford-White, Y.-C. Yang, L.-M. Zhu, E.W. Welbeck, X.-L. Yang, A novel fast disintegrating tablet fabricated by three-dimensional printing, Drug Dev Ind Pharm, 35 (2009) 1530-1536.

[27] W.E. Katstra, R.D. Palazzolo, C.W. Rowe, B. Giritlioglu, P. Teung, M.J. Cima, Oral dosage forms fabricated by Three Dimensional Printing ${ }^{\text {TM }}$, J Control Release, 66 (2000) 1-9.

[28] A. Aburub, D.S. Risley, D. Mishra, A critical evaluation of fasted state simulating gastric fluid (FaSSGF) that contains sodium lauryl sulfate and proposal of a modified recipe, Int J

[29] B. comission, British Pharmacopeia 2017, The British Pharmacopoeia Commission (BCP) Office, London, 2017.

[30] W.L. Hulse, J. Gray, R.T. Forbes, A discriminatory intrinsic dissolution study using UV area imaging analysis to gain additional insights into the dissolution behaviour of active pharmaceutical ingredients, Int J Pharm, 434 (2012) 133-139.

[31] J. Ostergaard, UV imaging in pharmaceutical analysis, J Pharm Biomed Anal, (2017).

[32] T.T. Kararli, Comparison of the Gastrointestinal Anatomy, Physiology, and Biochemistry of Humans and Commonly Used Laboratory-Animals, Biopharmaceutics \& Drug Disposition, 16 (1995) 351-380.

[33] M. Mori, Y. Shirai, Y. Uezono, T. Takahashi, Y. Nakamura, H. Makita, Y. Nakanishi, Y. Imasato, Influence of specific gravity and food on movement of granules in the gastrointestinal tract of rats, Chem Pharm Bull (Tokyo), 37 (1989) 738-741.

[34] E.L. McConnell, A.W. Basit, S. Murdan, Measurements of rat and mouse gastrointestinal $\mathrm{pH}$, fluid and lymphoid tissue, and implications for in-vivo experiments, J Pharm Pharmacol, 60 (2008) 63-70. 

equilibration of deuterium oxide (D2O) in gastrointestinal contents and the proportion of total body water (T.B.W.) in the gastrointestinal tract, J Clin Invest, 36 (1957) 289-296. [36] C. Tuleu, C. Andrieux, P. Boy, J.C. Chaumeil, Gastrointestinal transit of pellets in rats: effect of size and density, Int J Pharm, 180 (1999) 123-131. gastrointestinal pH profiles in normal ambulant human subjects, Gut, 29 (1988) 1035-1041. [38] M. Kyobula, A. Adedeji, M.R. Alexander, E. Saleh, R. Wildman, I. Ashcroft, P.R. Gellert, C.J. Roberts, 3D inkjet printing of tablets exploiting bespoke complex geometries for controlled and tuneable drug release, J Control Release, 261 (2017) 207-215. innovative approach to accelerating drug release from 3D printed tablets, J Control Release, 269 (2018) 355-363.

\section{List of Figures}

500

Figure 1. Schematic diagram of SDi2 instrument. LED's of different wavelength are em ployed to absorbance of the sample was recorded using an Actipix detector. The medium is pre-heated to $37^{\circ} \mathrm{C}$ before going through the Whole Dosage Flow Cell and is recirculated in a closed loop configuration.

Figure 2. Precision of 3D printing to control low dose sodium warfarin. (A) Images of warfarin loaded FDM 3D printed tablets with increasing dose, (B) Correlation between the theoretical volume and tablet mass, (C) warfarin dose accuracy in the 3D printed tablets and (D) correlation between theoretical volume and warfarin dose $(n=3, \pm S D)$.

Figure 3. Thermal analysis of Eudragit E based 3D printing filaments. (A) Thermal degradation profiles for Eudragit E, sodium warfarin, TCP, warfarin loaded filament and tablet, (B) DSC thermograph for warfarin loaded filament and tablet, (C) XRD spectra of Eudragit E, TCP, warfarin, and warfarin loaded filament and tablet.

Figure 4. In vitro release pattern of sodium warfarin from 3D printed tablets of different doses from a USPII dissolution test in modified FaSSGF $(p H 2.0)(n=3, \pm S D)$.

Figure 5. Changes in tablet height (A) and width (B) at $0,10,20,30,40,50$ and 60 min of the flow through dissolution test using Actipix SDI 2 dissolution imaging technology. (C) UV absorbance image following the illumination of follow cell containing warfarin 3D printed tablet at $255 \mathrm{~nm}$ wavelength. (D) Percentage sodium warfarin release from $3 D$ printed tablet during dissolution test $(n=3, \pm S D)$.

519 Figure 6. (A1) Rendered images and (A2) photographs of purpose designed 3D printed tablets for 520 oral gavage in rats, (B) Plasma concentration- time profile of warfarin following the oral dosing of 521200 or $400 \mu \mathrm{g}$ from (B) warfarin solution and (C) warfarin loaded 3D printed tablets to adult heathy 522 male Sprague-Dawley rats $(n=4)$, error bars \pm SD.

\section{$523 \quad$ List of tables}

524 Table 1. Summary of pharmacokinetic parameters of warfarin following oral gavage of 200 or $400 \mu g$ 525 from sodium warfarin solution and 3D printed tablets to adult heathy male Sprague-Dawley rats.

\section{Supplementary data}


527 Table 1S. Summary of length, width, height and volume of cuboid containing warfarin loaded 3D 528 printed tablets.

529 
530 Tailored on demand anti-coagulant dosing: an in vitro and in

531 vivo evaluation of 3D printed purpose-designed oral dosage

532 forms

533

534 Basel Arafat ${ }^{1,2}$, Nidal Qinna² Milena Cieszynska1, Robert T Forbes ${ }^{1}$, Mohamed A 535 Alhnan $^{1^{*}}$

536

$537{ }^{1}$ School of Pharmacy and Biomedical Sciences and ${ }^{2}$ School of Medicine, University of Central

538 Lancashire, Preston, Lancashire, UK.

5392 Faculty of Pharmacy and Medical Sciences, University of Petra, Amman, Jordan.

540

${ }_{541}$ Supplementary Data

542

543

544

545

546

*Corresponding author: MAlbedAlhnan@uclan.ac.uk

547

548

549

550 
551 Table 1S. Summary of length, width, height and volume of cuboid containing sodium warfarin loaded 552 3D printed tablets

553

\begin{tabular}{lllll}
\hline $\begin{array}{l}\text { Target dose } \\
(\boldsymbol{\mu g})\end{array}$ & Volume $\left(\mathrm{mm}^{3}\right)$ & $\mathbf{X}$ & $\mathbf{Y}$ & $\mathbf{Z}$ \\
$\mathbf{3 0 0}$ & 19.06 & 5.09 & 1.86 & 2.00 \\
\hline $\mathbf{5 0 0}$ & 40.74 & 6.55 & 2.40 & 2.58 \\
\hline $\mathbf{1 0 0 0}$ & 94.93 & 8.68 & 3.18 & 3.42 \\
\hline $\mathbf{1 5 0 0}$ & 149.12 & 10.09 & 3.69 & 3.98 \\
\hline $\mathbf{2 0 0 0}$ & 203.31 & 11.19 & 4.09 & 4.41 \\
\hline $\mathbf{2 5 0 0}$ & 257.51 & 12.10 & 4.43 & 4.77 \\
\hline $\mathbf{3 0 0 0}$ & 311.70 & 12.90 & 4.72 & 5.08 \\
\hline $\mathbf{4 0 0 0}$ & 420.08 & 14.24 & 5.21 & 5.61 \\
\hline $\mathbf{5 0 0 0}$ & 528.47 & 15.38 & 5.62 & 6.06 \\
\hline
\end{tabular}

554

555

556 